Int. J. Dev. Biol. 53: 525-533 (2009)

doi: $10.1387 / \mathrm{ijdb} .082720 \mathrm{yy}$

\title{
The mob as tumor suppressor (mats1) gene is required for growth control in developing zebrafish embryos
}

\author{
YUAN YUAN ${ }^{1}$, SHUO LIN ${ }^{1,2}$, ZUOYAN ZHU ${ }^{1}$, WENXIA ZHANG*,1 and ZHI-CHUN LAI*,1,3,4 \\ ${ }^{1}$ Center of Developmental Biology and Genetics, School of Life Sciences, Peking University, Beijing, China, \\ ${ }^{2}$ Department of Molecular, Cell and Developmental Biology, University of California, Los Angeles, CA USA, ${ }^{3}$ Department of Biology and \\ ${ }^{4}$ Department of Biochemistry and Molecular Biology, The Pennsylvania State University, University Park, PA USA
}

\begin{abstract}
The mob as tumor suppressor (mats) family genes are highly conserved in evolution. The Drosophila mats gene functions in the Hippo signaling pathway to control tissue growth by regulating cell proliferation and apoptosis. However, nothing is known about whether mats family genes are required for the normal development of vertebrates. Here we report that zebrafish has three mats family genes. Expression of mats 1 is maternally activated and continues during embryogenesis. Through a morpholino-based knockdown approach, we found that mats 1 is required for normal embryonic development. Reduction of mats 1 function caused developmental delay, a phenotype similar to that of Drosophila mats homozygous mutants. Both cell proliferation and apoptosis were defective in mats1 morphant embryos. Moreover, mats 1 morphant cells exhibited a growth advantage in chimeric embryos, similar to mats mutant cells in mosaic tissues in Drosophila. Therefore mats1 plays a critical role in regulating cell proliferation and apoptosis during early development in zebrafish, and the role of mats family genes in growth regulation is conserved in both invertebrates and vertebrates. This work shows that zebrafish can be a good model organism for further analysis of Hippo signaling pathway.
\end{abstract}

KEY WORDS: zebrafish, growth control, mob as tumor suppressor, hippo signaling

\section{Introduction}

Hippo (Hpo) signaling plays a crucial role in controlling cell proliferation and apoptosis, and disruption of this growth regulatory mechanism causes tissue overgrowth in Drosophila (reviewed in Hariharan and Bilder, 2006; Harvey and Tapon, 2007; Pan, 2007; Saucedo and Edgar, 2007). While Hpo signaling is mediated through several tumor suppressor proteins such as Hippo (Hpo) protein kinase to activate Warts (Wts)/Large tumor suppressor (Lats) protein kinase, a Mob family protein Mats (Mob as tumor suppressor) is critical for activating the catalytic activity of Wts kinase (Lai et al., 2005; Wei et al., 2007). Consequently, a growth-promoting transcription coactivator Yorkie (Yki) and the Drosophila ortholog of mammalian Yes-associated protein (YAP) are inhibited by Wts/Lats protein kinases via phosphorylation and cytoplasmic retention (Huang et al., 2005; Dong et al., 2007; Wei et al., 2007; Zhao et al., 2007; Hao et al., 2008). When Yki is present in the nucleus, the TEAD family transcription factor
Scalloped (Sd) is turned on to promote tissue growth by forming a complex with Yki to directly activate transcription of target genes such as the Drosophila inhibitor of apoptosis (diap1) gene (Wu et al., 2008; Zhang et al., 2008). Although the Hpo signaling pathway has been extensively studied in Drosophila, much less is known about its components and physiological function in vertebrates.

The first Mob family protein was discovered in yeast as "Mps one binder protein" and shown to be a binding partner as well as a coactivator of protein kinases of the Ndr (nuclear Dbf2-related) family in regulating mitotic exit and cytokinesis (reviewed in Hergovich et al., 2006b). Mob proteins also have been studied in fly and mammalian cells in recent years. In Drosophila, Mats (also called dMob1) was discovered in 2005 as a coactivator of an Ndr family serine/threonine protein kinase Wts to control cell proliferation and apoptosis (Justice et al., 1995; Xu et al., 1995; Lai et al.,

Abbreviations used in this paper: Mats, mob as tumor suppressor.

\footnotetext{
*Address correspondence to: Wenxia Zhang. College of Life Sciences, Peking University, Beijing, 100871, China. Fax: +86-10-6275-6185. e-mail: wxzhang@pku.edu.cn or Zhi-Chun Lai. Department of Biology, The Pennsylvania State University, 201 LSB, University Park, PA 16802, USA. Fax:+1-814-863-1357. e-mail: zcl1@psu.edu
}

Supplementary Material for this paper is available at: http://dx.doi.org/10.1387/ijdb.082723yy

Accepted: 15 July 2008. Published online: 20 January 2009. Edited by: Chrisopher Wylie.

ISSN: Online 1696-3547, Print 0214-6282

() 2009 UBC Press

Printed in Spain 
2005). Recently, Mats has been shown to be phosphorylated and activated by $\mathrm{Hpo} / \mathrm{Mst}$ protein kinases in both fly and human cells (Wei et al., 2007; Praskova et al., 2008). Interestingly, while loss of mats function causes tissue overgrowth in mosaic flies (Lai et al., 2005), mutants homozygous for mats are developmentally delayed and die at an early larval stage (He et al., 2005; Shimizu et al., 2008). Drosophila Mob family proteins also genetically interact with tricornered (trc), which is another Ndr family protein kinase in Drosophila and is required for the normal morphogenesis of a variety of polarized outgrowths (He et al., 2005). In human, LATS1 interacts with MATS/MOBKL1, and hLATS1 activation may be mediated through rapid recruitment to the plasma membrane by hMATS (Hergovich et al., 2005; Hergovich et al., 2006a). Functionally, hLATS1/hMATS complex appears to be required for cytokinesis and mitotic exit (Yang et al., 2004; Bothos et al., 2005). Although a human mats ortholog hMATS1 can rescue the lethality and tumor phenotypes of Drosophila mats mutants (Lai et al., 2005), nothing is known about the physiological function of mats family genes during vertebrates development.

We chose zebrafish to investigate the role of mats in vertebrate development, since zebrafish provides a genetic model system to study early development and cancer-related genes (Amaruda et al., 2002; Stern et al., 2003; Berghmans et al., 2005; Shepard et al., 2005). Two mats orthologs have been identified in zebrafish (Lai et al., 2005). Here, we report that the zebrafish genome has one more mats ortholog. We show that zebrafish mats 1 is maternally expressed and is also expressed throughout embryogenesis. Using a morpholino-based gene knockdown approach, we found that mats 1 is required for normal embryonic development, and is involved in regulating both cell proliferation and apoptosis. Similar to what was observed in Drosophila, mats 1 morphant cells seem to have a growth advantage over wild-type cells in chimeric zebrafish embryos. Our results suggest that growth regulatory properties of mats are conserved in vertebrates.

\section{Results}

\section{Three mats orthologs exist in zebrafish}

Through a phylogenetic analysis, two orthologs of the Drosophila mats gene have been identified in vertebrates (Lai et al., 2005). In zebrafish, mats 1 (also named mobkl1b for Mps One Binder kinase activator-like $1 \mathrm{~b}$ ) and mats2 (also named mobkl1a for Mps One Binder kinase activator-like 1a) genes encode protein products that share 85 and $88 \%$ identity with the Drosophila Mats protein, respectively (Lai et al., 2005; Supplementary Fig. S1A). Through synteny analysis, the arrangement of genes in the flanking regions of mats 1 and mats 2 was found to be highly conserved in zebrafish, mouse and human (Supplementary Fig. S1B and S1C). These results confirmed the orthologous relationships of mats 1 and mats 2 genes in these vertebrates. By searching the updated zebrafish genome database, we found that zebrafish has an additional mats ortholog, mats3 (also named mob4b), whose intron-exon structure is identical to other vertebrate mats genes while other mob family genes have distinct intron-exon structures (X. Ye and Z.-C. Lai, unpublished results). Similar to Mats1 and Mats2, the zebrafish Mats3 protein is $88 \%$ identical to Drosophila Mats. As mats3 is not found in other vertebrates, it is likely a product of gene
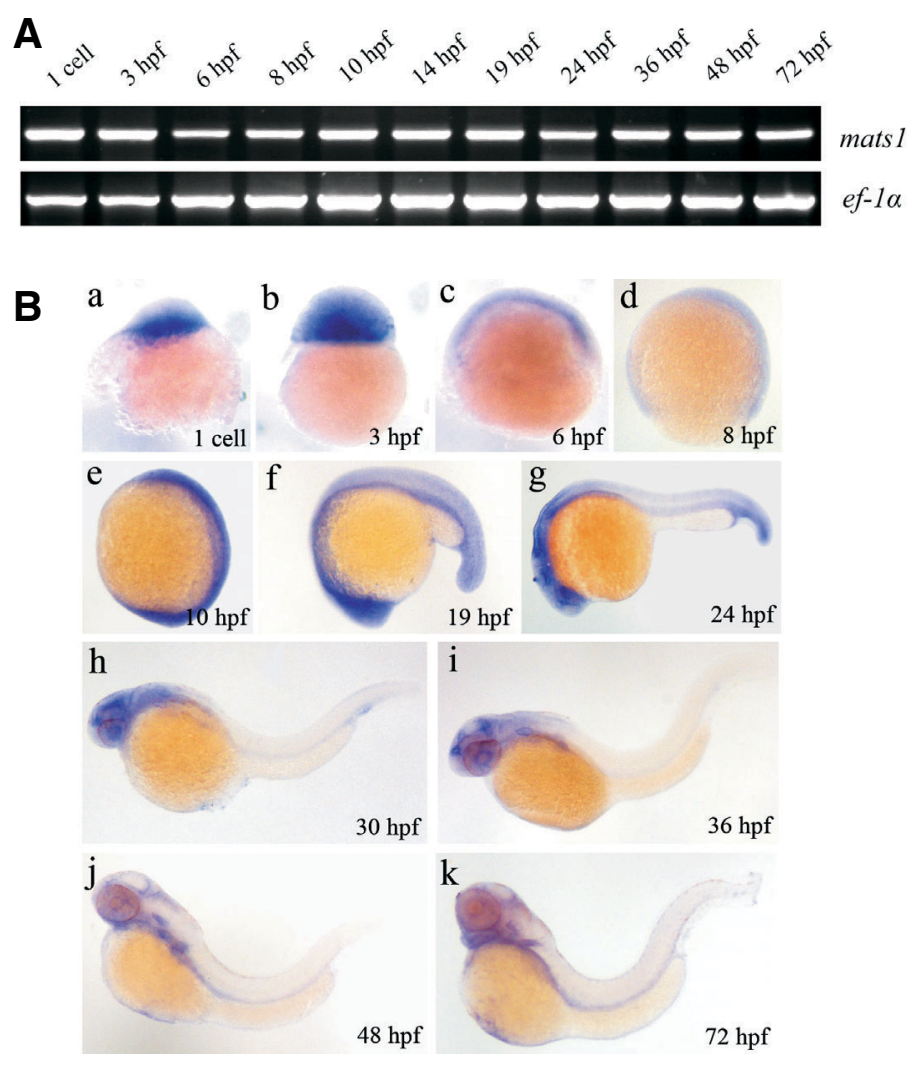

Fig. 1. mats 1 is expressed during zebrafish early development. (A) Temporal expression of mats 1 detected by RT-PCR. ef-1a is shown on the bottom panel as an internal control. (B) Expression of mats1 during early development detected by in situ hybridization. The animal pole is towards the top in (a-d). Anterior is towards the top and dorsal is towards right in (e). Anterior is towards left and dorsal towards top in (f-k). They are all showed by lateral view. Before $24 \mathrm{hpf}$, expression of mats 1 is ubiquitous. After 24hpf, mats1 expression was observed in the head region of the body.

duplications occurred after divergence of fish from other vertebrates. For clarity, the terms mats 1, mats 2 and mats 3 refer to the above genes are used throughout this paper. In this study we have focused on mats 1 to investigate its developmental role in zebrafish embryos.

\section{mats1 mRNA is maternally stored and expressed during early embryonic development}

To facilitate functional analysis of mats genes, expression of mats1 during early development was examined through RTPCR and in situ hybridization. RT-PCR results showed that mats 1 mRNA was detected at the one-cell stage of embryonic development (Figure 1A). Thus, mats 1 mRNA is maternally provided. Moreover, mats 1 was continuously expressed throughout the first three days after fertilization, with some reduction at 6 hours post fertilization (hpf) (Figure 1A). RNA whole-mount in situ hybridization confirmed this result, and provided information about the spatial distribution of mats 1 mRNA (Figure 1B). Before $24 \mathrm{hpf}$, mats 1 was broadly expressed in the embryo (Figure 1B, a-g). After $24 \mathrm{hpf}$, expression of mats 1 was stronger in the head than in the trunk (Figure 1B, h-k). This expression 
analysis suggests that mats 1 plays a role during embryonic development.

\section{mats 1 is required for normal embryonic development}

Morpholino-based antisense oligonucleotides provide an efficient and specific means to block protein translation in zebrafish embryos (Nasevicius and Ekker, 2000; Draper et al., 2001). To investigate the function of mats 1 during embryogenesis, a translation-blocking morpholino (MO1) and a splice-blocking morpholino (MO2) were designed to knock down mats 1 expression (Figure 2A). Interestingly, both MO1 and MO2 caused a phenotype with developmental delay. MO1 was less effective since only $20-30 \%$ of morphant embryos exhibited the delay phenotype. In contrast, MO2 was much more effective; over $70 \%$ of the morphant embryos showed the delay phenotype when injected with $8.5 \mathrm{ng}$ of mats $1 \mathrm{MO} 2$. Among these abnormal mats1 morphants, over $50 \%$ of them showed 16.5 hpf morphology, $20-30 \%$ with morphol- ogy between 16 and $18 \mathrm{hpf}$ stages, and 10-20\% with morphology between 18 and 20 hpf stages. This effect was concentrationdependent (data not shown). Consequently, mats1 MO2 was used throughout this study.

To determine the efficiency and specificity of mats 1 morpholino treatment, RT-PCR was done at both $10 \mathrm{hpf}$ and $24 \mathrm{hpf}$ with primers corresponding to exons flanking the MO2 target site (Figure 2A). We found that MO2 treatment caused $70-80 \%$ reduction of-mats 1 expression in embryos showing severe developmental delay (Figure $2 \mathrm{~A}$, lanes 4 and 8 ), whereas normallooking morphant embryos had less reduction of mats 1 expression (40-50\% of the wild-type level) (Figure 2A, lanes 3 and 7 ). While MO2 binding appears to block correct splicing of mats 1 transcript, no aberrant splicing was observed. It is possible that the splice-modified mats 1 mRNA cannot be exported and consequently degraded in the nucleus. As internal controls, expression of the two other mats orthologs mats2 and mats 3 was not affected
A

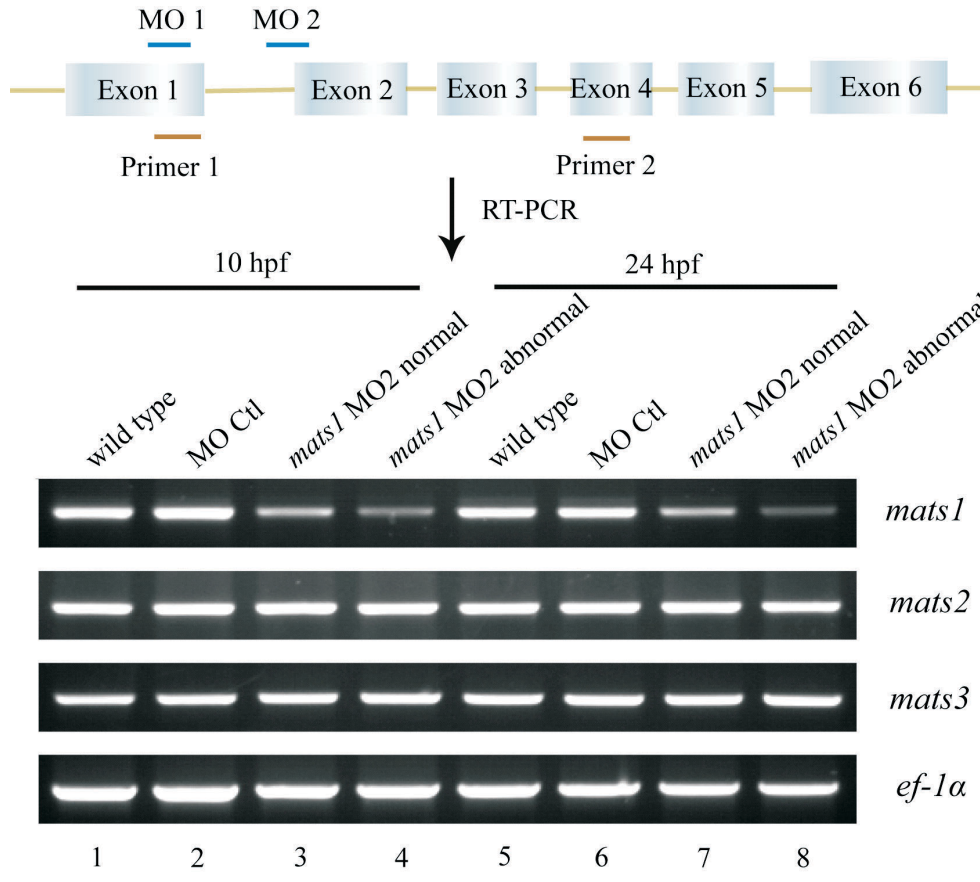

B

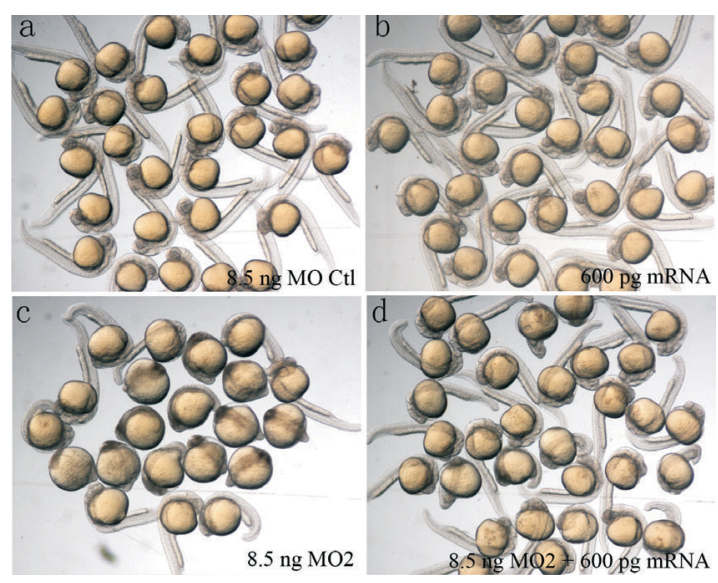

Fig. 2. Expression of mats 1 is reduced by morpholino treatment. (A) Location of mats 1 MOs and effect of mats 1 MO2 on mats1 mRNA levels. The schematic structure of mats1 gene is shown, and the size is not in scale. mats 1 MO1 binds to ATG site and mats 1 MO2 binds to the intron 1-exon2 boundary. RT-PCR was done with primers 1 and 2 to detect mats $1 \mathrm{mRNA}$ levels at $10 \mathrm{hpf}$ and 24 hpf. mats 1 MO2 morphants showing abnormal phenotype and normal phenotype were grouped separately. Expression of mats 1 was reduced in mats 1 MO2 morphants (lane 3-4 and lane 7-8) compared to wild-type (lane 1 and 5) and MO Ctl morphant (lane 2 and 6) embryos. Degree of the reduction was positively associated with severity of abnormal phenotype. As controls, mRNA levels of mats 2 and mats 3 were not affected. ef- $1 \alpha$ was used as an internal control. (B) Rescue of mats1 MO2-induced developmental delay phenotype by mats1 mRNA. (a) Embryos injected with $8.5 \mathrm{ng} \mathrm{MO} \mathrm{Ctl} \mathrm{as} \mathrm{a} \mathrm{control.} \mathrm{(b)} \mathrm{Embryos} \mathrm{injected} \mathrm{with}$ 600 pg mats1 mRNA exhibited normal phenotype. (c) Embryos injected with $8.5 \mathrm{ng}$ mats $1 \mathrm{MO} 2$ showed severe developmental delay. (d) Most embryos co-injected with 600 pg mats 1 mRNA and 8.5 ng mats1 MO2 showed normal or less severe abnormal phenotype. (C) Rescue of mats 1 morphants by mats 1 mRNA is dosage-dependent. At $24 \mathrm{hpf}$, only 19\% $(n=181)$ of the mats 1 MO2 morphant embryos were normal. However, co-injection of 300 pg mats1 mRNA with mats1 MO2 made $37 \%$ $(n=142)$ of the embryos to become normal. When 600 pg mats1 mRNA was coinjected, 55\% ( $n=196)$ of the embryos showed a normal phenotype. Although many remaining embryos still exhibited a developmental delay phenotype, the severity was decreased. Embryos injected with $8.5 \mathrm{ng} \mathrm{MO} \mathrm{Ctl}$ were used as a control $(n=134)$. All the statistical data included dead embryos. All the living embryos injected with $8.5 \mathrm{ng} \mathrm{MO} \mathrm{Ctl}$ were normal. Standard errors were shown by the error bars. 
by mats $1 \mathrm{MO} 2$ (Figure 2A). These results indicated that mats 1 mRNA levels can be effectively and specifically reduced by morpholino treatment.

Moreover, mats $1 \mathrm{mRNA}$ was co-injected with mats $1 \mathrm{MO} 2$ to test whether mats 1 mRNA is able to rescue the abnormal phenotypes induced by mats $1 \mathrm{MO} 2$. While injection of mats $1 \mathrm{mRNA}$
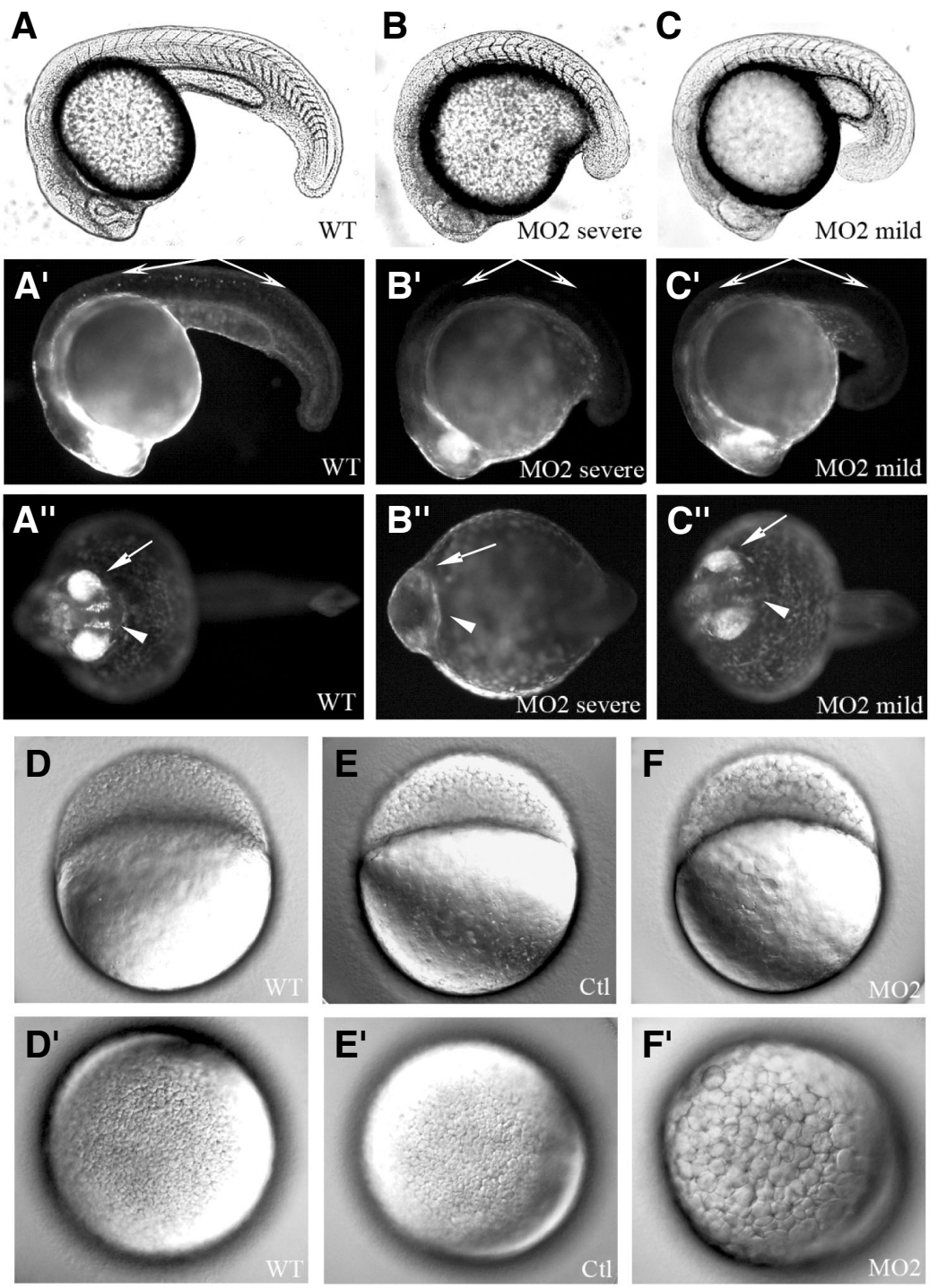

Fig. 3. The mats 1 gene is required for normal development of zebrafish embryos. (A-C") 24 hpf embryos of a transgenic line 1040 whose CNS is marked by GFP. Bright-field images in (A-C). Fluorescent images of the same embryos shown in ( $\mathbf{A}^{\prime} \mathbf{-} \mathbf{C}$ "). Lateral view in $\left(A-C^{\prime}\right)$. Ventral view in $\left(A^{\prime \prime}-C^{\prime \prime}\right)$. "Severe" represents mats 1 MO2 morphants which showed severe developmental delay, while "mild" represents those showed a weaker developmental delay phenotype. At 24 hpf, some neurons in trunk (indicate by long arrow) had already emerged in wild-type embryos, but they were not observed in mats 1 MO2 morphants. Eyes (indicated by short arrow) were either not visible (B') or less developed (C') in mats1 MO2 morphants. Similarly, the brain (indicate by arrow head) was less developed in mats1 MO2 morphants (B','C'). (D-F') Embryos at 3.5 hpf. mats1 MO2 morphants show developmental delay at very early stage. Lateral view with anterior towards left in (DF). Top view to see animal pole in ( $\left.\mathbf{D}^{\prime}-\mathbf{F}^{\prime}\right)$. mats1 MO2 morphant embryos had fewer but bigger cells (F, $\left.\mathbf{F}^{\prime}\right)$ compared to wild-type embryos (D, $\left.\mathbf{D}^{\prime}\right)$ and embryos injected with MO Ctl (E,E'), suggesting that mats1 MO2 morphant cells divided less than control cells during the same period of time. alone did not cause any abnormal phenotype (Figure 2B-b), coinjection of mats 1 mRNA with $\mathrm{MO} 2$ effectively rescued $\mathrm{MO} 2$ (Figure 2B, compare image (d) with (c)). With creased from $19 \%$ to $36 \%$ and $55 \%$, respectively (Figure $2 \mathrm{C}$ ). 1 nucleotides which can be recognized by mats $1 \mathrm{MO} 2$ still remained in the in vitro transcript mats $1 \mathrm{mRNA}$, titration of mats $1 \mathrm{MO} 2$ by mats 1 mRNA might exist. To further confirmed that the developmental delay phenotype was specifically caused by mats 1 knock down, a putative mats $1 \mathrm{MO} 2-$ binding defective (MO2-bd in short) mRNA in which 5 nucleotides in the mats $1 \mathrm{MO} 2$ binding region were mutated based on degeneracy of codons was also synthesized to do rescue experiment. With 20 pg MO2bd mRNA co-injection, proportion of normal-looking embryos increased from 19 to $65 \%$ (Supplementary Figure S2 compare A with $\mathrm{C}$ ). Consistently, while injected with $20 \mathrm{pg}$ MO2-bd mRNA alone, no abnormal phenotype was observed (Supplementary Figure S2B). It seemed that the putative MO2-bd mRNA can rescue the MO2 morphant embryos much more effectively than wild type mats $1 \mathrm{mRNA}$. One explanation is that wild type mats 1 but not the mutant mats 1 mRNA can be targeted by $\mathrm{MO2}$. These results support the idea that MO2induced abnormalities were due to the reduction of mats1 function.

Some morpholinos are known to activate the p53 pathway by an off-target effect (Robu et al., 2007). As mats1 morphant embryos exhibited elevated expression of $\Delta 113 p 53$ (a truncated version of $p 53$ ) and $p 21$ (a direct target of p53), we tested whether activation of p53 pathway contributes to the developmental defects of mats 1 morphants. To do this, we co-injected p53 MO with mats $1 \mathrm{MO} 2$ and confirmed that $p 53 \mathrm{MO}$ can effectively reduce $p 21$ and $\Delta 113 p 53$ ( $p 53 \mathrm{MO}$ binds to $p 53$ start codon, so it can block $p 53$ translation without affecting RNA expression of $p 53$ ) expression. However, mats1/p53 morphant embryos still exhibited the developmental delay phenotype (Supplementary Figure S3A). These results further support that reduction of mats 1 function disrupted normal embryogenesis.

Reduction of mats 1 expression and function causes developmental delay. At $24 \mathrm{hpf}$, delayed mats 1 morphants only had 14-22 somites just like 16-20 hpf wild-type embryos, whereas wild-type siblings had 26 somites. More than $50 \%$ of mats 1 morphant embryos that showed severe developmental delay phenotype only had less than 16 somites. The trunk of mats 1 morphants was shorter and more curved (Figure 3, AC). Given more time, mats 1 morphants developed more somites although their overall morphology was still abnormal. Development of the central nervous system was also delayed (Figure 3, A'-C' and A"-C'). Consistently, the head and eyes of mats 1 morphants usually were smaller. Assessment of marker genes like no tail, goosecoid, frb35, pax2, myoD showed that expression of these genes was delayed without changing their expression patterns (data not shown). The delayed phenotype was also found in mats 1 morphant embryos 
at earlier stages. At $10 \mathrm{hpf}$, epiboly of siblings injected with $\mathrm{MO}$ Ctl was already complete but mats 1 morphants showed only $50-90 \%$ epiboly (Figure 4K). At about $3 \mathrm{hpf}$, when wild-type siblings reached the 1000 cell stage, the cleaving morphant embryos had fewer but larger cells, demonstrating that they divided less often than their wild-type siblings (Figure 3, compare F-F' with D-D' and E-E'). Thus, reduction of mats 1 function impedes embryonic development.

Knockdown of mats1 function also reduced viability of the morphants. About $50-80 \%$ mats 1 morphants showing abnormalities survived five days post fertilization (dpf), while others died along the way. When wild-type embryos normally hatched from $48 \mathrm{hpf}$ to $72 \mathrm{hpf}$, the mats 1 morphants did not hatch from the chorion, and consequently, only survived up to 5 dpf before using up the yolk. Those that successfully hatched were unable to escape and swim away when touched. Instead, they could only circle at the same location, likely due to defects in their neural and muscular systems. Moreover, some mats 1 morphants also exhibited defects such as pericardial expansion, reduced number of otoliths, and decreased density of blood cells. Thus, mats 1 function appears to be required throughout development in many tissues.

\section{Defective cell proliferation in mats1 morphant embryos}

Our analysis has focused on growth defects of mats 1 morphant embryos. Because mats1 morphants had fewer cells than wild-type siblings at the same age, cell proliferation and/or apoptosis were likely aberrant due to the reduction of mats 1 function. To test this idea, we first determined whether cell proliferation in mats 1 morphant embryos was defective. For this purpose, we used BrdU staining to mark S-phase cells and phosphohistone $\mathrm{H} 3(\mathrm{PH} 3)$ antibody staining to mark M-phase cells. At $24 \mathrm{hpf}$, BrdU incorporation decreased in mats 1 morphant embryos (36/41) compared to wild-type siblings and siblings injected with MO Ctl (Figure 4, A-F). This reduction of S-phase cells was more evident in mats 1 morphant embryos showing severely delayed phenotype than normal-looking morphant embryos. The same experiment was repeated with $10 \mathrm{hpf}$ embryos, and the results were consistent with those of $24 \mathrm{hpf}$ embryos (Figure 4, G-K). Since mats1 morphant embryos were developmentally delayed, the decrease of S-phase cell number could be attributed to age differences between mats 1 morphant and control embryos. To test this, mats 1 morphant embryos were cultured for a few more hours until they reached the tail bud stage (equivalent to10 hpf in wild-type embryos at $28.5^{\circ} \mathrm{C}$ ). Embryos
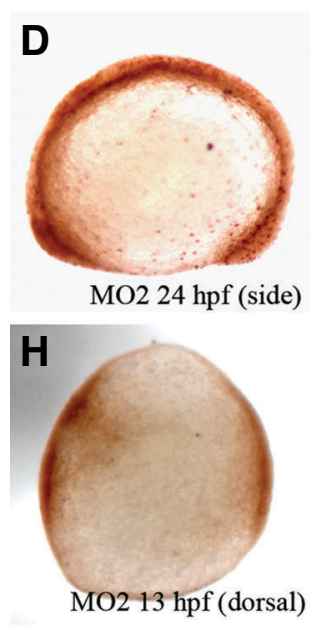
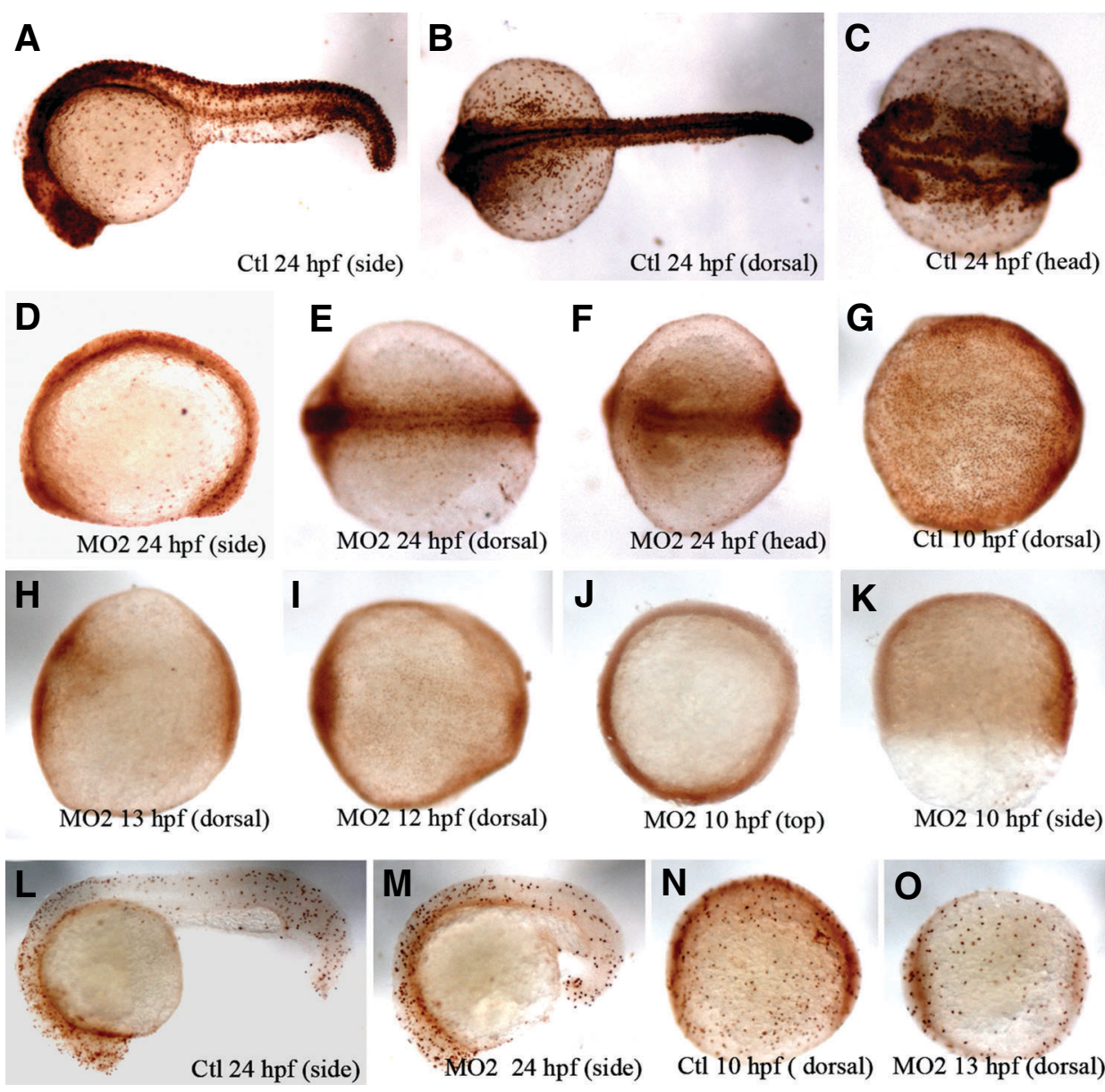

Fig. 4. Cell proliferation was decreased in mats 1 morphant embryos. (A-K) BrdU staining results at $24 \mathrm{hpf}$ and tail bud stage. At the $24 \mathrm{hpf}$ stage, BrdU levels were reduced in mats 1 morphant embryos 10 hpf (G), this process is not completed in mats 1 MO2 morphant embryos with the same age (J, K)

wo three more hours were needed for mats1 MO2 morphant embryos to reach tail bud stage antibody staining results at tail bud stage and $24 \mathrm{hpf}$ were consistent with BrdU staining, although the decreased degree of marked cell is not as distinctive as BrdU staining results. Anterior is towards left in all panels except for $(\mathbf{J}, \mathbf{K})$. Top view to see animal pole in $(\mathrm{J})$, animal pole is towards top in (K).

that showed severe developmental delay needed $13 \mathrm{~h}$ to reach this stage, while those that showed a mild phenotype needed 11$12 \mathrm{~h}$. Interestingly, these embryos still did not have the same number of S-phase cells as control embryos at $10 \mathrm{hpf}$ (Figure 4, compare $\mathrm{H}-\mathrm{I}$ with $\mathrm{G}$ ).

$\mathrm{PH} 3$ antibody staining was done to identify mitotic cells in embryos. At an early stage (10-13 hpf), the number of mitotic cells in severely delayed mats 1 morphant embryos was decreased compared with uninjected siblings and embryos injected with $\mathrm{MO}$ control (about $50 \%$ of control embryos) (Figure $4, \mathrm{~N}-\mathrm{O}$ ). But at 24 hpf, the PH3 staining results didn't show marked difference when comparing mats1 morphants with control embryos. The difference between BrdU and PH3 staining suggests that mats1 may be involved in cell cycle control. To further test this idea, fluorescence-activated cell sorting (FACS) analysis was done with mats 1 morphant and control embryos at $24 \mathrm{hpf}$ to see whether the ratios of cells at different phases of the cell cycle changed. We 

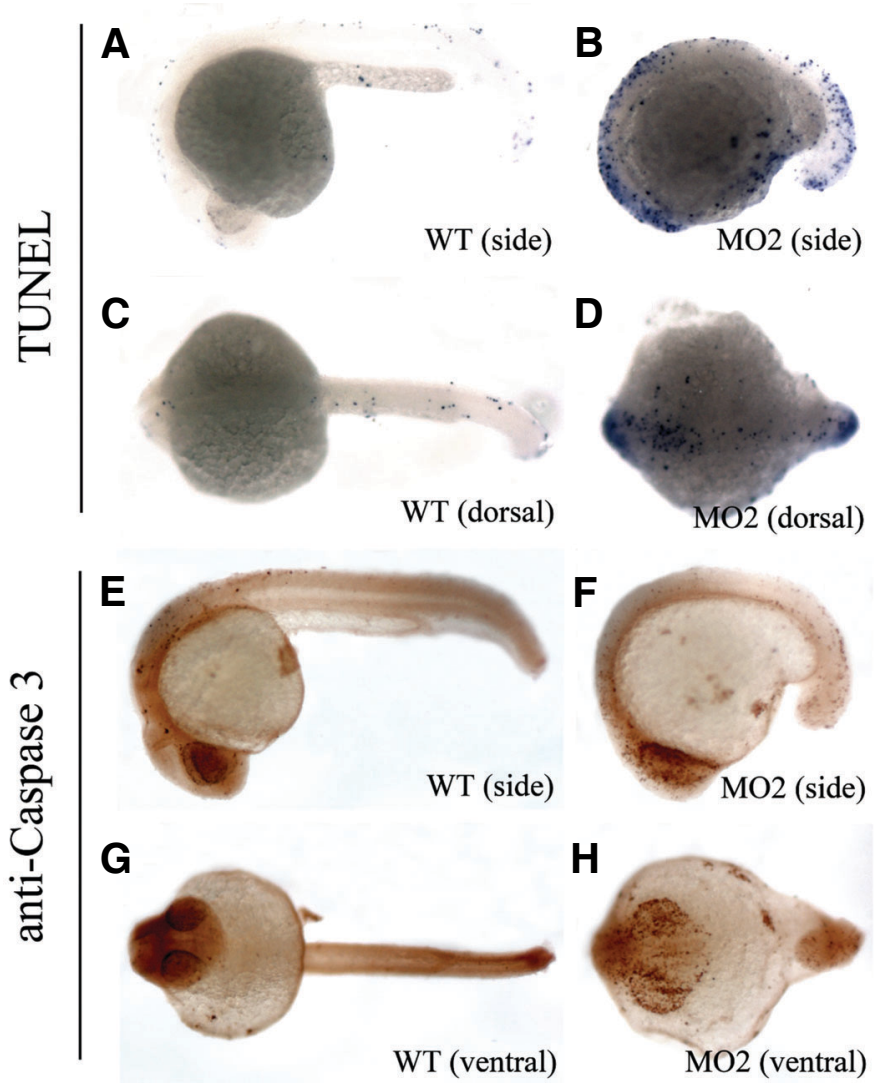

Fig. 5. Apoptosis was increased in mats 1 morphant embryos. (A-D) TUNEL staining results at $24 \mathrm{hpf}$ derived from wild type embryos $(\mathbf{A}, \mathbf{C})$ and MO2-treated embryos (B,D). (E-H) Cleaved Caspase 3 antibody staining results at the same developmental stage with wild type embryos $(\mathbf{E}, \mathbf{G})$ and MO2 treated embryos $(\mathbf{F}, \mathbf{H})$. Anterior is towards left in all panels. Both of TUNEL and cleaved Caspase 3 antibody-staining results showed that apoptosis increased in mats1 morphant embryos.

found that the ratio of cells at the M-phase in mats1 morphant embryos was more than that of the control embryos (13.33 $\pm 0.57^{\text {* }}$ vs. $9.60 \pm 0.20^{*}$, “*” represents standard error, t-test, $\left.p<0.005\right)$, while the ratio at S-phase didn't change much in mats 1 mrophant embryos compared to control embryos (29.68 $\pm 1.00^{*}$ vs. $30.82 \pm$ $0.74^{*}$, “*” represents standard error). These results indicate that loss of mats 1 function may have mitotic defects that cause the accumulation of mitotic cells.

\section{Apoptosis increased in mats1 morphant embryos}

To test whether mats 1 is involved in regulating cell death, the TUNEL assay was done at $24 \mathrm{hpf}$, when apoptosis normally occurs in developing embryos. Compared to control embryos (Figure 5, A and C), mats1 morphant embryos showed an increase in TUNEL-positive cells (29/38) (Figure 5, B and D). Thus, more cells died in mats 1 morphant embryos. The results are similar when coinjected with p53MO. Thirty-nine out of 46 mats $1 /$ p53 morphant embryos still exhibited excessive apoptosis with decreased signal in head region (Figure S3B), which are consistent with a former report (Robu et al., 2007).

To determine whether cell death in mats 1 morphant embryos is mediated through apoptosis, cleaved Caspase 3 antibody staining was done to specifically label apoptotic cells. The results of cleaved Caspase3 staining were consistent with the TUNEL results (Figure 5, E-H). Compared to control embryos (Figure 5, $\mathrm{E}$ and $\mathrm{G})$, mats 1 morphant embryos clearly exhibited increased apoptosis at $24 \mathrm{hpf}(50 / 50)$, mainly in the head and caudal parts (Figure 5, F and H). Thus, knockdown of mats 1 leads to increased cell death, and this occurs mainly through apoptosis.

\section{mats1 morphant cells have a growth advantage in chimeric zebrafish embryos}

Loss of mats function causes mutant cells to overproliferate in mosaic fruit flies (Lai et al., 2005). To determine how mats1deficient cells might behave when surrounded by normal cells in zebrafish, we carried out cell transplantation experiments to generate mats 1 chimeric embryos (Supplementary Figure S4). From three independent experiments, four hundred thirty one mats 1 morphant cells and 522 cells from embryos injected with MO Ctl were transplanted into more than 130 embryos at the 3-4 hpf stage. By $10 \mathrm{hpf}$, they had proliferated to generate 3,010 and 2,840 cells, respectively. Therefore, their respective proliferation index (PI) was 6.98 and 5.44 (t-test, $p<0.0001$; Table 1). From two other control experiments, the proliferation index of cells from embryos injected with FITC or Rhodamine were 5.84 and 5.59 respectively, similar to cells from embryos injected with MO Ctl (Table 1). Similar to control normal cells, mats 1 morphant cells distributed throughout the embryo. These results suggested that mats 1 morphant cells in chimeric embryos had a growth advantage and proliferated more than normal cells. Thus, the growth inhibitory activity of mats family genes appears to be conserved in zebrafish.

\section{Discussion}

All vertebrate Mats proteins share extremely high levels of sequence identity with Drosophila Mats. Yet functional significance of mats genes in vertebrate development has not been investigated. On the basis of functional analysis of mats in Drosophila (Lai et al., 2005; Wei et al., 2007; Shimizu et al., 2008), we hypothesized that zebrafish mats 1 plays a critical role during embryogenesis. Supporting this idea, mats1 is expressed in developing embryos. Using a morpholino-based loss-of-function analysis, we found that mats 1 plays a critical role in regulating cell proliferation and apoptosis in early embryos. Similar to Drosophila homozygous mats mutants (He et al., 2005; Shimizu et al., 2008), reduction of mats 1 expression caused severe develop-

TABLE 1

\section{MATS1 MORPHANT CELLS PROLIFERATE FASTER THAN WILD-TYPE CELLS IN CHIMERIC EMBRYOS}

\begin{tabular}{cccc} 
& N1 (total) & N2 (total) & PI on average \\
\hline mats1 MO2 & 431 & 3010 & $6.98 \pm 0.37^{\star}$ \\
MO Ctl & 522 & 2840 & $5.44 \pm 0.27^{\star}$ \\
FITC Ctl & 69 & 395 & $5.72 \pm 0.08^{\star}$ \\
Rhodamine Ctl & 50 & 282 & $5.64 \pm 0.13^{\star}$ \\
\hline
\end{tabular}

Proliferation index (PI, defined by N2/N1) of four types of cells. N1 refers to the cell number at 3-4 hpf, and N2 refers to the cell number at $10 \mathrm{hpf}$. mats $1 \mathrm{MO} 2$ :mats $1 \mathrm{MO} 2$ morphant cells. MO Ctl: cells from embryos injected with control MO. FITC Ctl: cells from embryos which were only injected with FITC. Rhodamine Ctl: cells from embryos which were only injected with Rhodamine.

"** represents standard error. It is calculated with average proliferation index derived from three independent transplantation experiments, and represents the deviation of $\mathrm{PI}$ on average. 
mental delay of the zebrafish morphant embryos, which exhibited reduced cell proliferation and increased apoptosis. This is the first time that a mob family gene is shown to be required for normal embryogenesis in vertebrates. Further studies shall reveal how Hippo signaling might function to control early development in vertebrate animals.

\section{Growth inhibitory role of mats appears to be conserved in zebrafish}

mats was first discovered in Drosophila as a tumor suppressor because loss of mats function caused tissue overgrowth in mosaic flies (Lai et al., 2005). In this study we have tested whether the growth inhibitory activity of mats family gene is conserved in vertebrates by generating chimeric zebrafish embryos through cell transplantation. Interestingly, chimeric analysis showed that mats 1 morphant cells proliferated faster than control normal cells, just like mats mutant cells in mosaic fly tissues. It is less likely that the increase of mats 1 morphant cell number was caused by inhibition of cell death, since cell death barely occurs before 10 hpf. Thus, this growth-inhibitory role of mats appears to be evolutionarily conserved between invertebrates and vertebrates. mats 1 morphant cells in chimeric embryos had a growth advantage likely through competition with neighboring wild-type cells. To acquire such a growth advantage, mats 1 morphant cells may need to be stimulated by surrounding wild-type cells, or capable of inhibiting growth of neighboring wild-type tissues. Although the mechanism by which mats 1 morphant cells acquire growth advantage in the context of chimeric embryos is presently unknown, cell-cell interaction should play a critical role in this process. A future challenge is to reveal the molecular basis of this intercellular interaction critical for tissue growth control during animal development.

\section{Materials and Methods}

\section{Animals}

The wild-type Tübingen strain or Tübingen/AB stain zebrafish and a transgenic line 1040 whose central nervous system is marked by GFP were used in this study. Zebrafish embryos were obtained by natural spawning, raised at $28.5^{\circ} \mathrm{C}\left( \pm 0.5^{\circ} \mathrm{C}\right)$ in Holtfreter's solution and the developmental stage determined as described by KIMMEL et al. (1995).

\section{Whole-mount in situ hybridization}

Digoxigenin-labeled antisense RNA probes were generated in vitro by using a zebrafish mats 1 full-length cDNA as template with T7 or SP6 RNA polymerase (Promega, Madison, WI). Whole-mount RNA in situ hybridizations were performed essentially as described in The Zebrafish Book (Westerfield et al., 1995) on embryos at the following developmental stages: 1 cell, 3 hours after fertilization (hpf), $6 \mathrm{hpf}, 8 \mathrm{hpf}, 10 \mathrm{hpf}$, 19 hpf, 24 hpf, 30 hpf, 36 hpf, 48 hpf and 72 hpf.

\section{In vitro mRNA synthesis}

Capped mRNAs were transcribed from linearized DNA using T7, T3 and SP6 RNA polymerase in vitro transcription kits according to the manufacturer's instruction (mMESSAGE, mMACHINE; Ambion). The pCMV-SPORT6 and pXT7 vectors were used. Moreover, site-directed mutagenesis through fusion PCR was done to make five silent nucleotide replacements within the MO2-binding site of mats 1 to convert 5'CCATTTGATTTCAGC GGA AAC CGT T-3' to 5'- CCATTTGATTTCAGt GGg AAt aGg T -3'. This mutant mats1 still encodes a wild-type Mats protein product and its mRNA was synthesized in vitro as described above for the rescue experiment.

\section{Microinjection}

Capped mRNAs and morpholinos were dissolved and diluted in nuclease-free water to an appropriate concentration. They were injected into 1- to 2-cell-stage embryos in yolk. The amount of injection was determined by measuring the volume of liquid injected into a $1-\mu$ l capillary glass (34 mm long) using a ruler, and volume per microinjection was thereby calculated.

\section{Morpholino design and phenotypic analysis}

One translation-blocking morpholino (MO1) and one splice-blocking morpholino (MO2) of mats 1 were synthesized by Open Biosystems, Inc. (Huntsville, AL). The sequence of MO1 is:

5'-TTCCGAATAAGAAACTCATCTCCGC-3', which corresponds to the start codon region, and the sequence of $M O 2$ is:

5'-AACGGTTTCCGCTGAAATCAAATGG-3', which corresponds to the putative intron1-exon2 boundary of mats 1 .

A translation blocking morpholino targeting p53 from Gene Tools (Philomathe, OR) was used to eliminate $p 53$ dependent off-target effect, its sequence is 5'-GCGCCATTGCTTTGCAAGAATTG-3'. A standard control morpholino oligo (MO Ctl) from Gene Tools (Philomathe, OR) was used as control. Its sequence is $5^{\prime}$-CCTCTTACCTCAGTTACAATTTATA3 '.

Injected embryos were cultured in Holtfreter's solution at $28.5^{\circ} \mathrm{C}$ $\left( \pm 0.5^{\circ} \mathrm{C}\right)$. An embryo was considered abnormal if it showed less than $90 \%$ epiboly when its siblings injected with the same mount of MO Ctl exhibited complete epiboly at the $10 \mathrm{hpf}$ stage, or if it appeared younger than $20 \mathrm{hpf}$ (defined by its overall look and somite number) compared to its siblings injected with the same mount of MO Ctl at the $24 \mathrm{hpf}$ stage.

\section{Reverse Transcription-Polymerase Chain Reaction (RT-PCR) and quantification}

For detection of expression pattern of mats 1 during early development, total RNA was extracted from wild-type embryos at various stages with Trizol reagent (Invitrogen, Carlsbad, CA). To detect splice-blocking variants of mats 1 mRNA with RT-PCR, total RNA was extracted from the morphant and control embryos (injected with $\mathrm{MO} \mathrm{Ctl}$ at the same quantity and uninjected wild-type siblings) at $10 \mathrm{hpf}$ and $24 \mathrm{hpf}$. Reverse transcription was carried out with random nonamer by using M-MLV reverse transcriptase (Promega, Madison, WI). Two rounds of PCR were performed. Primers used for detection of zebrafish mats 1 mRNA [GenBank: BC045979] were mats 1 forward (base pairs 273-297): 5'GAAGAAGAAGGACAAGCGGAGATG -3', and mats1 reverse (base pairs 758-734): 5'-CAGACGCTTCAGGATCGTTTTAGC-3'. The product size of mats1 RT-PCR is $485 \mathrm{bp}$. Zebrafish mats2 [GenBank: BC045952] and mats3 [GenBank: NM_214783] mRNA levels were determined with following primers: mats2 forward (base pairs 1-20): 5'AGAAGTTTTCCACGGGCAGG-3', and mats2 reverse (base pairs 393374): 5'-GCAGCTTTCCTCAGTGCAGA-3', the product size of mats2 is $393 \mathrm{bp}$; mats3 forward (base pairs 105-126): 5'-AAGCCGAAGAAGAATA TTCCTG-3', mats3 reverse (base pairs 612-591): 5'AAGAGGTGTTGAGGTGAGCTTC -3', and the product size of mats 3 is 508 bp. Zebrafish elongation factor-1 $\alpha(e f-1 \alpha)$ [GenBank NM_131263] was used as an internal control. The ef- $1 \alpha$ primers were ef- $1 \alpha$ forward (base pairs 496-516): 5'-TCACCCTGGGAGTGAAACAGC-3', and ef-1 $\alpha$ reverse (base pairs 1188-1168): 5'-ACTTGCAGGCGATGTGAGCAG-3'. The product size of ef-1 $\alpha$ is $692 \mathrm{bp}$.

The quantification of RT-PCR bands was done with BandScan 5.0.

\section{BrdU staining}

BrdU (5-bromo-2-deoxyuridine) (Roche Diagnostics, Mannheim, Germany) staining was performed to label S-phase cells. It was done essentially according to the protocol in Methods in Cell Biology (Detrich 
et al., 2004). Embryos were dechorionated and chilled 15 minutes on ice in Holtfreter's solution, then placed in cold $10 \mathrm{mM} \mathrm{BrdU} / 15 \%$ Dimethylsulfoxide (DMSO) in Holtfreter's solution and chilled on ice for 20 minutes to allow uptake of BrdU. Then embryos were changed into warm Holtfreter's solution and incubated exactly 5 minutes at $28.5^{\circ} \mathrm{C}$. They were fixed 2 hours at room temperature in PFA (4\% paraformaldehyde buffered with $1 \times$ PBS), dehydrated in graded methanol:PBS series $(1: 3,1: 1,3: 1)$ and preserved in methanol at $-20^{\circ} \mathrm{C}$ at least overnight. Rehydrated in graded methanol:PBST [1xPBS with $0.1 \%(\mathrm{v} / \mathrm{v})$ Tween-20] series $(3: 1,1: 1,1: 3)$ for 5 minutes each, followed by in PBST twice, 5 minutes each. Embryos were digested in $10 \mu \mathrm{g} / \mathrm{ml}$ proteinase $\mathrm{K}$ for 10 minutes, washed in PBST for 5 minutes, and refixed in PFA for no more than 20 minutes, followed by 3 times quick washes in $\mathrm{H}_{2} \mathrm{O}$ with $0.1 \%(\mathrm{v} / \mathrm{v})$ Tween-20 and 2 times in $2 \mathrm{~N} \mathrm{HCl}$. Embryos were incubated 1 hour in $2 \mathrm{~N} \mathrm{HCl}$ to denature the labeled DNA to expose the BrdU epitope, rinsed several times in PBST to bring the $\mathrm{pH}$ back up to approximately 7 before adding blocking solution, and then blocked for at least 30 minutes in blocking solution (1\% DMSO + $0.1 \%$ Tween- $20+1 \%$ BSA $+2 \%$ serum in PBS). After that, embryos were incubated in monoclonal anti-BrdU antibody (Zhong Shan Jin Qiao) at a dilution of $1: 100$ in blocking solution overnight at $4{ }^{\circ} \mathrm{C}$. Subsequently, embryos were washed in PBST five times, 10 minutes each, and incubated in horseradish peroxidase-conjugated anti-mouse secondary antibody about 2 hours at room temperature. Finally, embryos were washed in PBST five times, 10 minutes each. Color reaction was developed with diaminobenzidine.

\section{PH3 and cleaved Caspase 3 antibody staining}

Mitotic cells were stained with a rat polyclonal anti phosphorylated histone $\mathrm{H} 3$ antibody from Upstate Biotechnology (Charlottesville, VA). It was performed essentially as described in The Zebrafish Book (Westerfield et al., 1995), with a dilution of 1:1000, and the horseradish peroxidasecoupled secondary antibody was diluted 1:250. Cleaved caspase 3 antibody staining was performed to detect apoptotic cells. The method is basically the same with $\mathrm{PH} 3$ staining, and the cleaved Caspase-3 (Asp175) antibody from Cell Signaling Technology, Inc. (Beverly, MA) was diluted 1:100 and the horseradish peroxidase-coupled secondary antibody was diluted 1:250.

\section{TUNEL staining}

For detection of cell death, terminal desoxynucleotidyl transferase mediated biotinylated UTP nick end labeling (TUNEL) was performed, using the In situ Cell Death Detection Kit (Roche Diagnostics, Mannheim, Germany). Embryos were dechorionated and fixed overnight at $4{ }^{\circ} \mathrm{C}$ in PFA, dehydrated in a graded ethanol:PBS series $(1: 3,1: 1,3: 1)$, then preserved in ethanol at $-20^{\circ} \mathrm{C}$ for at least 30 minutes. Embryos were rehydrated in a graded ethanol:PBST series $(3: 1,1: 1,1: 3)$ for 5 minutes each, washed in PBST for 5 minutes, then digested in proteinase $\mathrm{K}$ (10 $\mu \mathrm{g} / \mathrm{ml}$ ) at room temperature for 10 minutes. After being washed twice in PBST, embryos were postfixed in PFA for 20 minutes at room temperature. With 5 times washes in PBST, 5 minutes each, embryos were postfixed for 10 minutes at $-20^{\circ} \mathrm{C}$ with pre chilled ethanol:acetic acid (2:1). After being washed in PBST three times, 5 minutes each at room temperature, embryos were used for TUNEL assay according to manufacture's instruction.

\section{Cell transplantation}

Eggs were collected and raised in Holtfreter's solution with 50 units penicillin and $50 \mu \mathrm{g}$ streptomycin per $\mathrm{ml}$ (Gibco). Eggs that were used as donors were microinjected with Fluorescein isothiocyanate-dextran (FTIC) (Sigma Chemical Co., St. Louis, MO) or tetramethylrhodamine-dextran (TMR) (Invitrogen) (5\% in nuclease-free water) or one combination of mats $1 \mathrm{MO} 2$ or MO Ctl with one of these two dyes. Cell transplantation began at the $1000-2000$ cells stage. Since mats 1 MO2 could cause developmental delay started before the 1000 cell stage, embryos injected with mats $1 \mathrm{MO} 2$ were raised at $28.5^{\circ} \mathrm{C}\left( \pm 0.5^{\circ} \mathrm{C}\right)$ while the sibling embryos injected with $\mathrm{MO} \mathrm{Ctl} \mathrm{and} \mathrm{embryos} \mathrm{without} \mathrm{injection} \mathrm{were} \mathrm{raised} \mathrm{at} 25.5^{\circ} \mathrm{C}$ $\left( \pm 0.5{ }^{\circ} \mathrm{C}\right)$ to adjust their development stage. The temperature was calculated using the formula described in (Kimmel et al., 1995). To maximally eliminate the difference of proliferation ability among cells from different location, all the transplanted cells were fetched in the center of the deep layers of injected embryos. One to five transplanted cells from each donor embryos were loaded by suction, and then were injected among the deep cells at the center of the same wild-type sibling at the same stage, without damaging the yolk cell. The numbers of each marked cells were counted (record as N1) under fluorescent microscope after cell transplantation was done (usually about 1 hour from the first transplant was done) one by one in the order of transplantation, and counted again at about $10 \mathrm{hpf}$ (record as N2). N2 divided with N1 is the index of cell proliferation ability.

\section{Imaging}

Images of zebrafish embryos were acquired by using an Imager Z1 or Stemi 2000-C microscope equipped with an AxioCam digital camera and AxioVission software (Zeiss, Oberkochen, Germany), and then edited with PhotoShop CS2 9.0 (Adobe Systems, San Jose, CA).

\section{Acknowledgement}

We would like to thank Qichang Fan, Bo Zhang, Chuanmao Zhang and Shiqiang Wang at Peking University, Feng Shao at the National Institute of Biological Science, Beijing, Anming Meng at Tsinghua University for discussion, reagents, zebrafish lines and apparatus support. We thank Xing Ma and Yongfei Yang for participation in part of the project, and Li-Lun Ho for help order morpholinos. We also thank lain Bruce for revising manuscript. This work was supported by the National Natural Science Foundation of China (Grant No. 30270186 and No. 30510403188) and by a grant to Z.-C.L. from Pennsylvania Formula Tobacco Settlement Funds.

\section{References}

AMATRUDA, J. F., SHEPARD, J. L., STERN, H. M. and ZON, L. I. (2002). Zebrafish as a cancer model system. Cancer Cell 1: 229-231.

BERGHMANS, S., MURPHEY, R. D., WIENHOLDS, E., NEUBERG, D. and KUTOK, J. L. (2005). tp53 mutant zebrafish develop alignant peripheral nerve sheath tumors. Proc Natl Acad Sci USA 102: 407-412.

BOTHOS, J., TUTTLE, R. L., OTTEY, M., LUCA, F. C. and HALAZONETIS, T. D. (2005). Human LATS1 is a mitotic exit network kinase. Cancer Res 65: 65686575 .

DETRICH, H. W., III, WESTERFIELD, M. and ZON, L. I. (2004). The Zebrafish: Cellular and Developmental Biology. Elsevier Academic Press. 2nd edition

DONG, J., FELDMANN, G., HUANG, J., WU, S., ZHANG, N., COMERFORD, S.A., GAYYED, M.F., ANDERS R.A., MAITRA, A. and PAN, D. (2007). Elucidation of a universal size-control mechanism in Drosophila and mammals. Cell 130: 1120-1133.

DRAPER, B. W., MORCOS, P. A. and KIMMEL, C. B. (2001). Inhibition of zebrafish fgf8 pre- mRNA splicing with morpholino oligos: a qantifiable method for gene knockdown. Genesis 20: 154-156.

HAO, Y., CHUN, A., CHEUNG, K., RASHIDI, B. and YANG, X. (2008). Tumor suppressor LATS1 is a negative regulator of oncogene YAP. J Biol Chem 283: 5496-5509.

HARIHARAN, I. K. and BILDER, D. (2006). Regulation of imaginal disc growth by tumor-suppressor genes in Drosophila. Annu Rev Genet 40: 335-361.

HARVEY, K. and TAPON, N. (2007). The Salvador-Warts-Hippo pathway - an emerging tumour-suppressor network. Nat Rev Cancer 7: 182-191.

HE, Y., EMOTO, K., FANG, X., REN, N., TIAN, X. and JAN, Y.N. (2005). Drosophila Mob family proteins interact with the related tricornered (Trc) and warts (Wts) kinases. Mol Biol Cell 16: 4139-4152.

HERGOVICH, A., BICHSEL, S. J. and HEMMINGS, B. A. (2005). Human NDR kinases are rapidly activated by $\mathrm{MOB}$ proteins through recruitment to the 
plasma membrane and phosphorylation. Mol Cell Biol 25: 8259-8272.

HERGOVICH, A., SCHMITZ, D. and HEMMINGS, B. A. (2006a). The human tumour suppressor LATS1 is activated by human MOB1 at the membrane. Biochem Biophys Res Commun 345: 50-58.

HERGOVICH, A., STEGERT, M. R, SCHMITZ, D. and HEMMINGS, B. A. (2006b). NDR kinase regulate essential cell processes from yeast to humans. Nat. Rev. Mol. Cell Biol 7: 253-264.

HUANG J., WU, S., BARRERA, J., MATTHEWS, K. and PAN, D. (2005). The Hippo signaling pathway coordinately regulates cell proliferation and apoptosis by inactivating Yorkie, the Drosophila Homolog of YAP. Cell 122: 421-434.

JUSTICE, R. W., ZILIAN, O., WOODS, D. F., NOLL, M. and BRYANT, P. J. (1995). The Drosophila tumor suppressor gene warts encodes a homolog of human myotonic dystrophy kinase and is required for the control of cell shape and proliferation. Genes Dev 9: 534-546.

KIMMEL, C. B., BALLARD, W. W., KIMMEL, S. R., ULLMANN, B. and SCHILLING, T. F. (1995). Stages of embryonic development of the zebrafish. Dev Dyn 203: 253-310.

LAI, Z.C., WEI, X., SHIMIZU, T., RAMOS, E., ROHRBAUGH M., NIKOLAIDIS, N., Ho, L.L. and Li, Y. (2005). Control of cell proliferation and apoptosis by mob as tumor suppressor, mats. Cell 120: 675-685.

NASEVICIUS, A. and EKKER, S. C. (2000). Effective targeted gene «knockdown» in zebrafish. Nat Genet 26: 216-220.

PAN, D. (2007). Hippo signaling in organ size control. Genes Dev. 21: 886-897.

PRASKOVA, M., XIA, F. and AVRUCH, J. (2008). MOBKL1A/MOBKL1B phosphorylation by Mst1 and Mst2 inhibits cell proliferation. Curr Biol 18: 311-321.

ROBU, M. E., LARSON, J. D., NASEVICIUS, A., BEIRAGHI, S., BRENNER, C., FARBER, S.A. and EKKER, S.C. (2007). p53 activation by knockdown technologies. PLoS Genet 3: e78.

SAUCEDO, L. J. and EDGAR, B. A. (2007). Filling out the Hippo pathway. Nat Rev Mol Cell Biol 8: 613-621.
SHEPARD, J. L., AMATRUDA, J. F., STERN, H. M., SUBRAMANIAN, A., FINKELSTEIN, D., ZIAI, J., FINLEY, K.R., PFAFF, K.L., HERSEY, C., ZHOU, Y. et al. (2005). A zebrafish bmyb mutation causes genome instability and increased cancer susceptibility. Proc Natl Acad Sci USA 102: 13194-13199.

SHIMIZU, T., HO, L.L. and LAI, Z.C. (2008). The mob as tumor suppressorgene is essential for early development and regulates tissue growth in Drosophila. Genetics 178:957-965.

STERN, H. M. and ZON, L. I. (2003). Cancer genetics and drug discovery in the zebrafish. Nat Rev Cancer 3:1-7.

WEI, X., SHIMIZU, T. and LAI, Z. C. (2007). Mob as tumor suppressor is activated by Hippo kinase in growth inhibition in Drosophila. EMBO J 26: 1772-1781.

WESTERFIELD, M. (1995). The zebrafish book. Univ of Oregon Press, Eugene, OR. 3rd Edition.

WU, S., LIU, Y., ZHENG, Y., DONG, J. and PAN, D. (2008). The TEAD/TEF family protein Scalloped mediates transcriptional output of the Hippo growth-regulatory pathway. Dev Cell 14: 388-398.

XU, T., WANG, W., ZHANG, S., STEWART, R.A. and YU, W. (1995). Identifying tumor suppressors in genetic mosaics: the Drosophila lats gene encodes a putative protein kinase. Development 121: 1053-1063.

YANG, X., YU, K., HAO, Y., LI, D.M., STEWART, R., INSOGNA, K.L. and XU, T. (2004). LATS1 tumour suppressor affects cytokinesis by inhibiting LIMK1. Nat Cell Biol 6: 609-617.

ZHANG, L., REN, F., ZHANG, Q., CHEN, Y., WANG, B. and JIANG, J. (2008). The TEAD/TEF family of transcription factor Scalloped mediates Hippo signaling in organ size control. Dev Cell 14: 377-387.

ZHAO, B., WEI, X., LI, W., UDAN, R.S., YANG, Q., KIM, J., XIE, J., IKENOUE, T., YU, J., LI, L. et al. (2007). Inactivation of YAP oncoprotein by the Hippo pathway is involved in cell contact inhibition and tissue growth control. Genes Dev 21: 2747 -2461. 


\section{Further Related Reading, published previously in the Int. J. Dev. Biol.}

See our recent Special Issue Fertilization, in honor of David L. Garbers and edited by Paul M. Wassarman and Victor D. Vacquier at: http://www.ijdb.ehu.es/web/contents.php?vol=52\&issue=5-6

Expression of zinc transporter family genes in Dictyostelium Nobuya Sunaga, Meri Monna, Nao Shimada, Mai Tsukamoto and Takefumi Kawata Int. J. Dev. Biol. (2008) 52: 377-381

Analysis of a homologue of the adducin head gene which is a potential target for the Dictyostelium STAT protein Dd-STATa Ryota Aoshima, Rieko Hiraoka, Nao Shimada and Takefumi Kawata

Int. J. Dev. Biol. (2006) 50: 523-532

Identification of new modes of Dd-STATa regulation of gene expression in Dictyostelium by in situ hybridisation Nao Shimada, Mineko Maeda, Hideko Urushiharaand Takefumi Kawata

Int. J. Dev. Biol. (2004) 48: 679-682

Expression and role of adenylyl cyclases during late development in Dictyostelium discoideum

E Alvarez-Curto, ME Meima, P Schaap

Int. J. Dev. Biol. (2001) 45: S147-S148

The MADS-box transcription factor SRFA regulates different aspects of Dictyostelium discoideum development

N Moreno, JJ Vicente, R Escalante, L Sastre

Int. J. Dev. Biol. (2001) 45: S117-S118

Cell cycle phase, cellular Ca2+ and development in Dictyostelium discoideum. M Azhar, P K Kennady, G Pande, M Espiritu, W Holloman, D Brazill, R H Gomer and V Nanjundiah

Int. J. Dev. Biol. (2001) 45: 405-414

Dictyostelium discoideum: a model system for differentiation and patterning.

$R$ Escalante and J J Vicente

Int. J. Dev. Biol. (2000) 44: 819-835

Cell-cell signaling and adhesion in phagocytosis and early development of Dictyostelium.

E Bracco, B Pergolizzi, B Peracino, E Ponte, A Balbo, A Mai, A Ceccarelli and S Bozzaro Int. J. Dev. Biol. (2000) 44: 733-742

2006 ISI **Impact Factor $=3.577^{* *}$

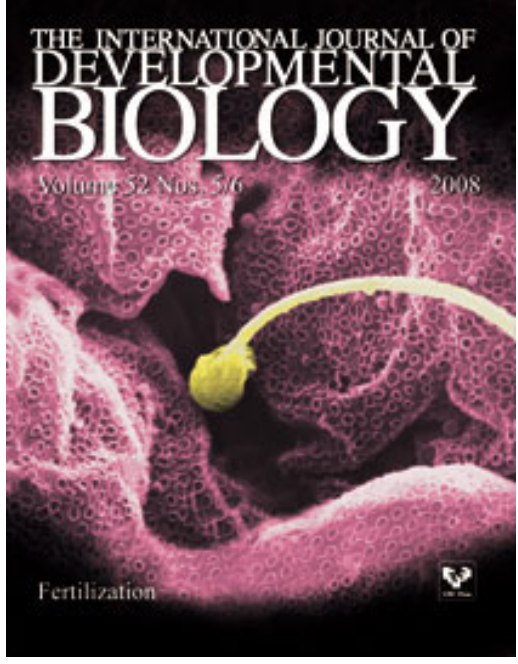

